\title{
Erratum: Single-band upconversion nanoprobes for multiplexed simultaneous in situ molecular mapping of cancer biomarkers
}

\author{
Lei Zhou, Rui Wang, Chi Yao, Xiaomin Li, Chengli Wang, Xiaoyan Zhang, Congjian Xu, Aijun Zeng, \\ Dongyuan Zhao \& Fan Zhang
}

Nature Communications 6:6938 doi: 10.1038/ncomms7938 (2015); Published 24 April 2015; Updated 12 Jun 2015

This Article contains errors in the author affiliation 1 and in Figs 2 and 6. The correct details for affiliation 1 are given below:

${ }^{1}$ Department of Chemistry and Laboratory of Advanced Materials, iChEm (Collaborative Innovation Center of Chemistry for Energy Materials), State Key Laboratory of Molecular Engineering of Polymers, Fudan University, Shanghai 200433, China.

In Fig. $2 \mathrm{~g}$, the emission spectrum was inadvertently duplicated from Fig. $2 \mathrm{c}$ during the production process. An error was also introduced in the transition represented in Fig. 2l. This panel represents the ${ }^{4} \mathrm{~F}_{9 / 2}-{ }^{4} \mathrm{I}_{15 / 2}$ transition. Furthermore, a wrong scale bar definition was provided in the figure caption. The scale bars represent $100 \mathrm{~nm}$. In Fig. 6a, the 'PR' and 'ER' labeling of MCF-7 and MDA-MB-231 cell images were inadvertently switched during manuscript preparation. The blue fluorescence of sb-UCNPs-Anti-ER should have been labelled 'ER', and the green fluorescence of sb-UCNPs-Anti-PR should have been labelled 'PR'. Corrected versions of both figures appear below. 

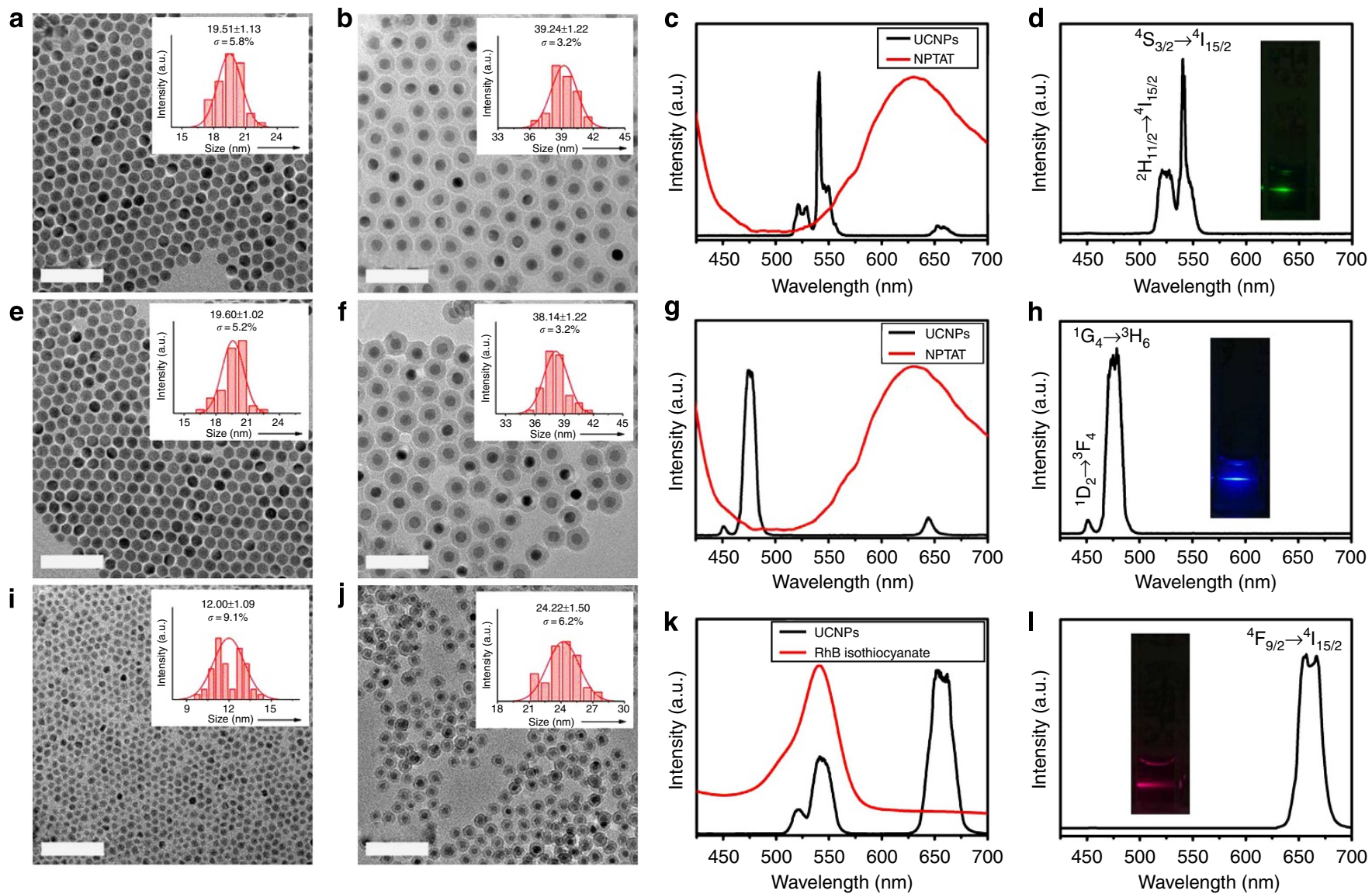

Figure 2 | Fabrication and properties of single-band upconversion nanoprobes. TEM images and size distributions of (a) $\beta-\mathrm{NaGdF}_{4}: 20 \% \mathrm{Yb}, 2 \%$ Er@NaGdF 4 nanocrystals, (b) green emission sb-UCNPs ( $\beta-\mathrm{NaGdF}_{4}: 20 \%$ Yb, 2\%Er@NaGdF $@ @ \mathrm{SiO}_{2} @ N P T A T-\mathrm{doped}_{\mathrm{SiO}}$ ), (e) $\beta-\mathrm{NaGdF}$ :20\% Yb, 0.2\%

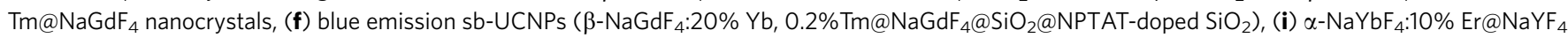
nanocrystals and (j) red emission sb-UCNPs $\left(\mathrm{NaYbF}_{4}: 10 \% \mathrm{Er}_{\mathrm{N} N \mathrm{NaF}} @ \mathrm{SiO}_{2} @\right.$ rhodamine B isothiocyanate-doped $\left.\mathrm{SiO}_{2}\right)$. Upconversion photoluminescence spectra of the green, blue and red sb-UCNPs $(\mathbf{d}, \mathbf{h}, \mathbf{I})$ and original upconversion nanocrystals measured in water and cyclohexane, respectively (c,g,k) (insets: corresponding photoluminescent photos of the colloidal solutions under continuous wavelength $980-\mathrm{nm}$ laser excitation). Scale bars, $100 \mathrm{~nm}$.

a
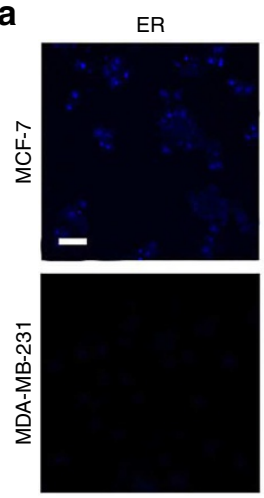

d
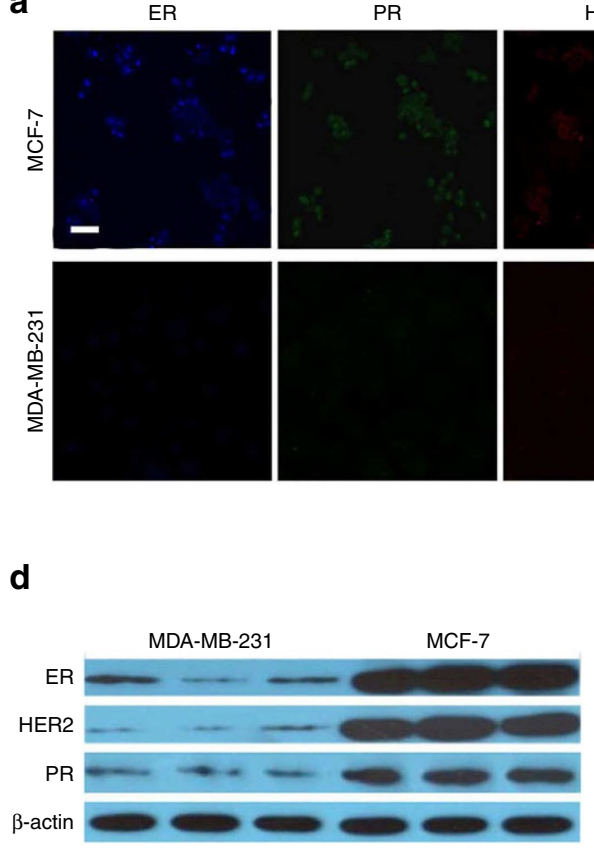

HER2
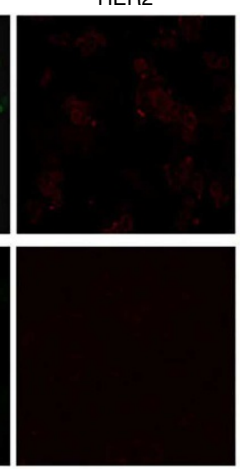

e

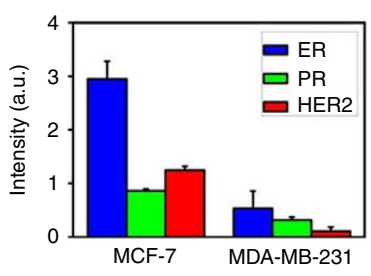

b
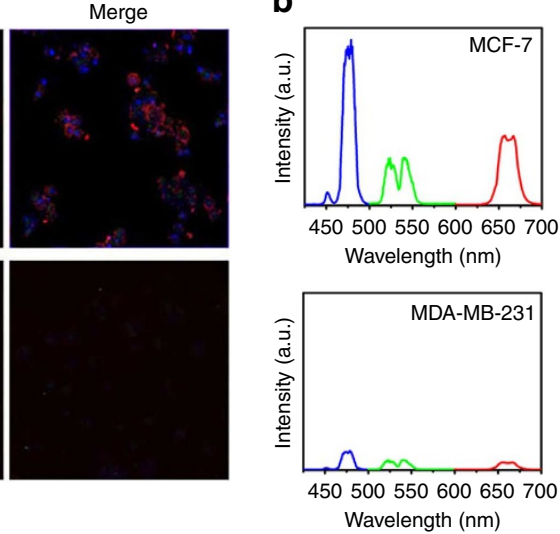

f c
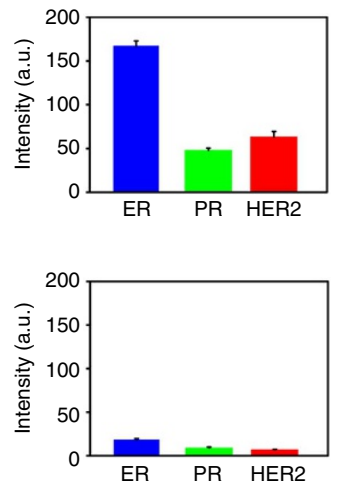

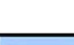

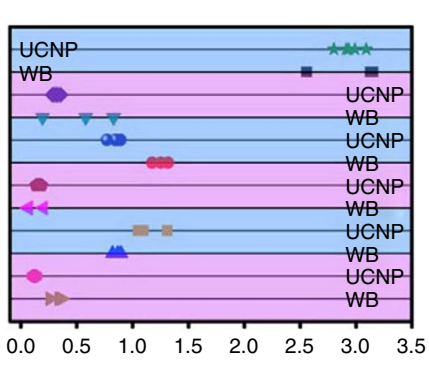

Intensity (a.u.) 